\title{
Evaluation of Gynogenic Responsiveness and Pollen Viability of Selfed Doubled Haploid Onion Lines and Chromosome Doubling via Somatic Regeneration
}

\author{
Marijana Jakše, Pablo Hirschegger, and Borut Bohanec ${ }^{1}$ \\ Department of Agronomy, Biotechnical Faculty, University of Ljubljana, Jamnikarjeva 101, \\ 1000 Ljubljana, Slovenia \\ Michael J. Havey \\ Department of Horticulture, Agricultural Research Service, U.S. Department of Agriculture, \\ University of Wisconsin, 1575 Linden Drive, Madison, WI 53706
}

\begin{abstract}
Additional INDEX words. Allium cepa, inheritance, inbreeding depression, homozygosity, male fertility
Abstract. Although haploid induction has been used in onions (Allium cepa L.) for over 20 years, several obstacles limit its use in plant breeding programs. To address these limitations, we evaluated the responsiveness of doubled haploid (DH) lines and their selfed progenies, an alternative protocol for chromosome doubling using somatic regeneration of haploid lines, and pollen viability of DH lines. Twenty-one DH lines were self pollinated and tested for haploid induction in the second generation. Among the DH lines, 18 lines showed an average of $20 \%$ decrease in gynogenic responsiveness compared with the original lines, while three lines registered an average increase of $5.7 \%$. Using a two-step induction/regeneration procedure, 8,589 somatic regenerants were obtained from 16,170 flower buds from haploid plants, and shoot culture was established. A more laborious procedure using extraction of ovaries in the regeneration stage was found equal to flower bud culture. Chromosome doubling via somatic regeneration was found to be $83 \%$ and $100 \%$ efficient when the source material was haploid or mixoploid, respectively. Based on the results achieved in this and previous studies, an alternative protocol for chromosome doubling of gynogenic haploids is proposed.
\end{abstract}

Haploid induction via unpollinated flowers or ovaries in onion has been practiced for 20 years, since the discovery that haploid plants can be obtained via gynogenesis (Muren, 1989). Following this major breakthrough, much effort has been made to improve the protocol efficiency, mainly by testing different media compositions (e.g., Campion et al., 1992; Jakše et al., 1996; Martínez et al., 2000; Ponce et al., 2006).

Although this procedure has become an integral part of many onion breeding programs, several obstacles still restrict its efficiency. Critical parameters include the role of genotype, the heritability of high gynogenic responsiveness, the chromosome doubling efficiency, and the male fertility of doubled haploid lines.

Genotype was recognized early as one of the most important factors affecting gynogenic haploid production in onion (Campion and Schiavi, 1994; Bohanec et al., 2003). The response of nonselected genotypes is highly variable; for instance, the haploid induction rate of 39 tested European, Japanese, and U.S. accessions ranged from 0 to 23 embryos per 100 flowers (Bohanec and Jakše, 1999). Within 22 European and U.S. cultivars, it ranged from 0 to 17 embryos per 100 flowers (Geoffriau et al.,

Received for publication 24 Nov. 2009. Accepted for publication 11 Jan. 2010. This work was financially supported by research grant P4-0077 from the Slovenian Research Agency.

We thank Viktorija Dolenc, Valerija Plestenjak, and Jože Godeša for their dedication in maintaining the onion collection.

Names are necessary to report factually on available data; however, the U.S. Department of Agriculture (USDA) neither guarantees nor warrants the standard of the product, and the use of the name by the USDA implies no approval of the product to the exclusion of others that may also be suitable.

${ }^{1}$ Corresponding author. E-mail: borut.bohanec@bf.uni-lj.si 1997b), and in 10 Polish cultivars, it ranged from 0 to 10 embryos per 100 flowers in which $30 \%$ of them were completely recalcitrant (Michalik et al., 2000). Because no efficient protocol had been found to induce haploid regenerants from recalcitrant genotypes, hybridization between responsive and nonresponsive lines was proposed to transmit gynogenic ability to the progeny (Bohanec et al., 2003).

Thus far, it is unclear whether the progeny of DH lines show high or low gynogenic haploid production. The first study that evaluated the gynogenic potential of $\mathrm{DH}$ lines was based on the evaluation of only three selfed progenies originated from cultivars Erso 1, Morgana, and Soriso (Javornik et al., 1998). The responsiveness was, on average, very low in two lines, 0.3 and 2.4 embryos per 100 flowers, while the third one was highly responsive, yielding 118.3 embryos per 100 flowers. The most responsive individual plant produced 196.5 embryos per 100 flowers. These findings clearly showed that not all DH regenerants possess genes that favor gynogenic responsiveness. Therefore, a second cycle of gynogenesis is needed to characterize the responsiveness of each DH line.

Because spontaneous chromosome doubling in onion haploids is usually below $10 \%$, an efficient chromosome doubling method becomes a key prerequisite for their use in breeding programs (Bohanec, 2002). Several approaches have been studied, based mainly on various antimitotic treatments. Treatment of in vitro-grown haploid split bulbils with colchicine was first studied in onion by Campion et al. (1995). Replacement of colchicine by oryzalin and trifluralin (Geoffriau et al., 1997a; Grzebelus and Adamus, 2004) did not significantly improve the doubling efficiency. When gynogenic embryos were treated, instead of shoot or in vitro-induced bulbils, a significant increase 
in doubling efficiency (about 35\%) was reported; particularly when amiprofos-methyl (APM) was used as the antimitotic substance (Jakše et al., 2003; Grzebelus and Adamus, 2004). Alan et al. (2004) reported that the use of APM in gynogenic embryos has several drawbacks, such as high mortality of treated explants, the inability to determine whether the diploid plants are of spontaneous or induced origin, and the loss of spontaneous diploid plants due to cytotoxicity or polyploidization. Instead, they proposed the use of APM or oryzalin in whole basal explants as alternatives to colchicine.

Alternative approaches have been proposed to overcome the problems associated with use of antimitotic drugs. Stöldt (1994) proposed that in vitro tissue culture could be used to induce spontaneous separation of chimeric tissues. Keller and Korzum (1996) speculated that diploid segments are present within mixoploid inflorescences and might result in normal flowers capable of self-pollination and production of homozygous DH seeds. Alan et al. (2007) induced somatic regeneration from flower buds of haploid plants and suggested that this method might be promising because diploid plants were obtained from all genotypes tested. However, the tested somatic regeneration protocol yielded only 5.6 somatic regenerants per 100 flowers, and the overall doubling efficiency was estimated on a low number of regenerants, making the interpretation of those results very preliminary.

Inbreeding depression is a significant problem in onion (Villanueva-Mosqueda and Havey, 2001). It affects plant growth, bulb size, number of inflorescences (Erickson and Gabelman, 1954), sugar content and potassium concentration of flower nectar (Hagler, 1990; Lederhouse et al., 1968), and pollen viability (Nye and Waters, 1971; Ockendon and Gates, 1976). In general, onion inbred lines are only partially homozygous because only a limited number of selfing cycles are performed. Deleterious genes present in heterozygous populations are fully expressed in DH lines. The strong selection pressure present at the in vitro regeneration stage eliminates genotypes with major phenotypic aberrations, enabling the formation of vigorous DH plants with apparently normal phenotype despite their complete homozygosity (Bohanec, 2002). However, selection pressure at the regeneration and plantlet establishment phases is related only to the genes regulating vegetative growth, while no selection pressure is exerted on characters related to flowering. Fertile DH plants are used in breeding programs, but evaluation of male fertility among regenerants has not been studied in detail.

The main goal of this study was to overcome some critical points related to the use of $\mathrm{DH}$ technology in plant breeding. The specific objectives of this work were to compare the gynogenic responsiveness of selfed progenies from DH lines relative to their original donor lines, to evaluate an integrated approach to inducing fertile $\mathrm{DH}$ lines by means of somatic regeneration of haploid or mixoploid plants, and to measure pollen viability as an estimation of male fertility of DH lines.

\section{Materials and Methods}

Plant materials. Individual plants from the inbred longday onion lines B 2923 B, B 0223 B, MSU 2935 B, populations Timor 135, PI 233189, and the hybrid B 1717 B × B2923 B served as donors for the first cycle of gynogenesis in 1996, 1999, and 2001. For simplicity, this group will be further referred as "donors." After chromosome doubling, DH plants were self-pollinated in 2003 and bulbs were obtained in 2004 from their seeds. Finally, umbels obtained in May 2005 served as source material for the second cycle gynogenesis.

Gynogenic regenerants obtained after the second cycle of gynogenesis were allowed to produce bulbs that were replanted in 2007 and allowed to flower. These umbels served as source material for the somatic regeneration experiment. Some regenerants obtained in previous experiments were also selected.

The source material used for the assessment of pollen viability was selected from DH plants obtained via gynogenesis from 1996 to 2002.

GYNOGENIC INDUCTION. Individual flower buds were cut periodically before dehiscence from May to June. Flowers were dissected and sterilized for $10 \mathrm{~min}$ in $16.6 \mathrm{~g} \cdot \mathrm{L}^{-1}$ dichloroisocyanuric acid disodium salt (Sigma-Aldrich, St. Louis) with the addition of a few drops of Tween 20 (Sigma-Aldrich). After three rinses in sterile water, 30 flowers were cultured in $90-\mathrm{mm}$ (in diameter) petri dishes. The basal medium consisted of BDS macro- and microelements and vitamins (Dunstan and Short, 1977) (Duchefa Biochemie, Haarlem, The Netherlands), 500 $\mathrm{mg} \cdot \mathrm{L}^{-1}$ inositol (Sigma-Aldrich), and $200 \mathrm{mg} \cdot \mathrm{L}^{-1}$ proline (Duchefa Biochemie). The basal medium was supplemented with $100 \mathrm{~g} \cdot \mathrm{L}^{-1}$ sucrose (Duchefa Biochemie), $7.5 \mathrm{~g} \cdot \mathrm{L}^{-1}$ Daishin agar (Duchefa Biochemie), $2 \mathrm{mg} \cdot \mathrm{L}^{-1}$ 2,4-dichlorophenoxyacetic acid (2,4-D; BDH Chemicals, Poole, England), and $2 \mathrm{mg} \cdot \mathrm{L}^{-1}$ of 6-benzylaminopurine (BAP; Sigma-Aldrich) for induction of gynogenic response. Petri dishes were sealed with parafilm (Pechiney Plastic Packaging, Chicago) and cultured in growing chambers. Environmental conditions in the chambers were 21 to $23{ }^{\circ} \mathrm{C}, 16 / 8 \mathrm{~h}$ (light/dark) light periods and photosynthetic photon flux $(P P F)$ of $80 \mu \mathrm{mol} \cdot \mathrm{m}^{-2} \cdot \mathrm{s}^{-1}$. An elongation medium composed of half-strength BDS medium supplemented with $15 \mathrm{~g} \cdot \mathrm{L}^{-1}$ glucose (Duchefa Biochemie) and $7.5 \mathrm{~g} \cdot \mathrm{L}^{-1}$ Daishin agar was used for sprouted gynogenic embryos. Before autoclaving, all media were adjusted to $\mathrm{pH}$ 6.0.

Somatic Regeneration. Flowers were cultured as described for gynogenic induction. The protocol followed that developed for somatic regeneration of onion diploid tissues (Luthar and Bohanec, 1999). The basal medium composition was the same as described for gynogenic induction. Two somatic induction procedures were studied: 1) flowers buds were cultured on induction medium and were then transferred to the differentiation one; and 2) ovaries were extracted from flowers buds before being transferred to differentiation medium. The induction period was $7 \mathrm{~d}$ long in both procedures. The induction medium was composed of basal medium supplemented with $2 \mathrm{mg} \cdot \mathrm{L}^{-1}$ 2,4-D, $2 \mathrm{mg} \cdot \mathrm{L}^{-1} \mathrm{BAP}, 2.5 \mathrm{~g} \cdot \mathrm{L}^{-1}$ gellan gum (SigmaAldrich), and $100 \mathrm{~g} \cdot \mathrm{L}^{-1}$ sucrose. The differentiation medium was composed of basal medium supplemented with $2 \mathrm{mg} \cdot \mathrm{L}^{-1}$ tidiazuron (TDZ; Duchefa Biochemie), $2.5 \mathrm{~g} \cdot \mathrm{L}^{-1}$ gellan gum, and $100 \mathrm{~g} \cdot \mathrm{L}^{-1}$ sucrose. After somatic regeneration, shoot clumps were transferred to elongation medium, composed of half-strength BDS, $15 \mathrm{~g} \cdot \mathrm{L}^{-1}$ glucose, and $8 \mathrm{~g} \cdot \mathrm{L}^{-1}$ Daishin agar. Before autoclaving, all media were adjusted to $\mathrm{pH} 6.0$.

Ploidy measurement. The ploidy level of mature plants obtained via gynogenesis was determined by flow cytometry as described earlier (Bohanec and Jakše, 1999). Briefly, young leaves of acclimatized plants were chopped in $1 \mathrm{~mL}$ of $0.1 \mathrm{M}$ citric acid (Sigma-Aldrich) containing 0.5\% Tween 20. A 3fold volume of dye solution containing $5.25 \mathrm{mg} \cdot \mathrm{mL}^{-1}$ 4',6diamidino-2-phenylindole (Partec, Münster, Germany) in $0.4 \mathrm{M}$ disodium hydrogen phosphate (Sigma-Aldrich) was added to 
the filtered suspension and analyzed using a Partec PAS flow cytometer. For the somatic experiment, ploidy was measured in flower buds of donor plants before plating, and following somatic regeneration, in young green leaves formed during the micropropagation stage.

Measurement of POLLEN ViabiLITy. Material for estimation of male fertility was obtained from DH lines. From each DH line, three open flowers were collected, and from each flower, three anthers were dispersed in a drop of $1 \%$ staining solution of aceto-carmine (Sigma-Aldrich) on a microscope slide. For each sample, 10 photographs were taken using a Carl Zeiss Jena Lumar stereo microscope (Carl Zeiss Lichtmikroskopie, Jena, Germany) at 100× magnification and a camera (Coolpix 990; Nikon, Tokyo). Dark red-stained pollen grains were counted as viable.

Data ANALYSES. In the gynogenic experiment, all available flowers were plated. The experimental unit was a petri dish containing 30 flower buds, and the number of repetitions ranged from two to 26 according to umbel number and size. In the somatic experiment, two treatments (flower buds and ovaries) with 10 repetitions each (less in case of contamination) were studied. The number of emerged gynogenic embryos or somatic regenerants per plated flower was scored weekly. Collected data were used to perform non-parametric analyses due to the lack of normality of the data sets. The Kruskal-Wallis test was used to determine whether there were statistically significant differences among mean regeneration rates of the studied genotypes in gynogenic and somatic experiments. In the so- matic experiment, flower bud and ovary culture were considered as treatments. The Mann-Whitney test was used to determine whether there were statistically significant differences between the effects of the different treatments and between the effects of the ploidy levels of the donor plants. All analyses were performed in SPSS Statistics (version 17.0; SPSS, Chicago).

\section{Results and Discussion}

Gynogenic Responsiveness OF SELFEd DH Lines. The gynogenic responsiveness of $\mathrm{S}_{1}$ progenies from $21 \mathrm{DH}$ lines and that of their original donor lines was compared (Table 1). In the first induction cycle, 6,613 flower buds were plated from 21 donor plants derived from the six onion donors. In the second induction cycle, a total of 19,455 flower buds were plated from $59 \mathrm{DH}$ plants derived from 21 selfed DH lines.

The responsiveness of the donors ranged from 0.03 to 0.82 embryos per flower plated, while the responsiveness of the selfed DH lines ranged from 0 to 0.63 embryos per flower plated. For 18 of 21 DH lines, a decrease in gynogenic responsiveness was observed compared with the responsiveness of their donors (mean -0.205 per flower plated). In only three lines was a slight increase in gynogenic responsiveness noted (mean +0.057 per flower plated). The Kruskal-Wallis nonparametric test revealed highly significant differences among the mean responsiveness of donors, as well as among the selfed DH lines $(P<0.001)$.

Table 1. Mean gynogenic embryo (GE) responsiveness of onion donors (first generation GE) and their selfed doubled haploid (DH) progenies.

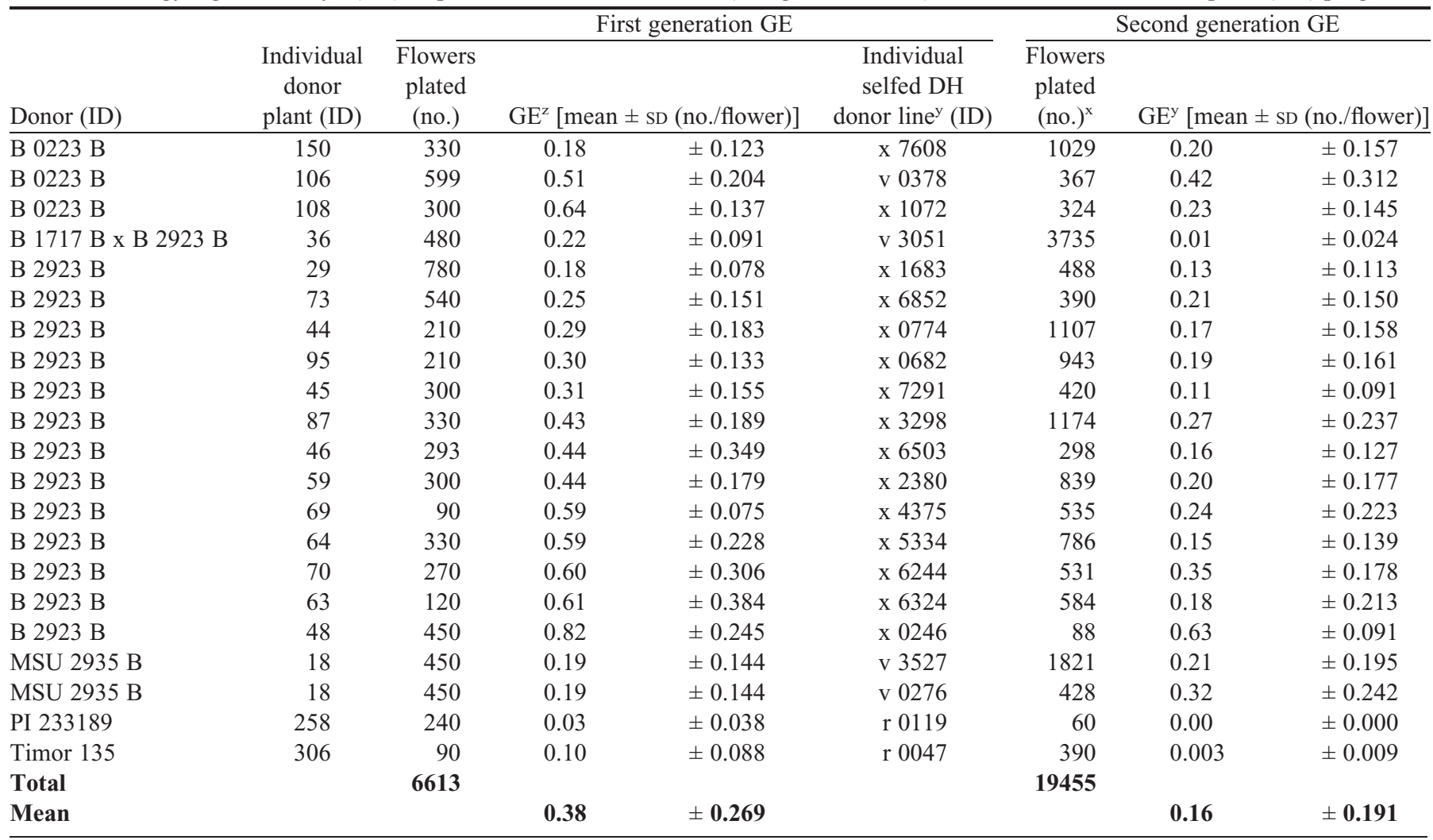

$\overline{{ }^{\mathrm{Z}} \text { Kruskal-Wallis non-parametric test found highly statistically significant differences among the mean responsiveness of donors, as well as among }}$ the selfed DH donor lines $(P<0.001)$.

${ }^{y}$ Selfed DH donor lines were not obtained in the same year.

${ }^{x}$ Flowers were obtained from one to five individual plants grown from seeds of selfed DH donor lines. 


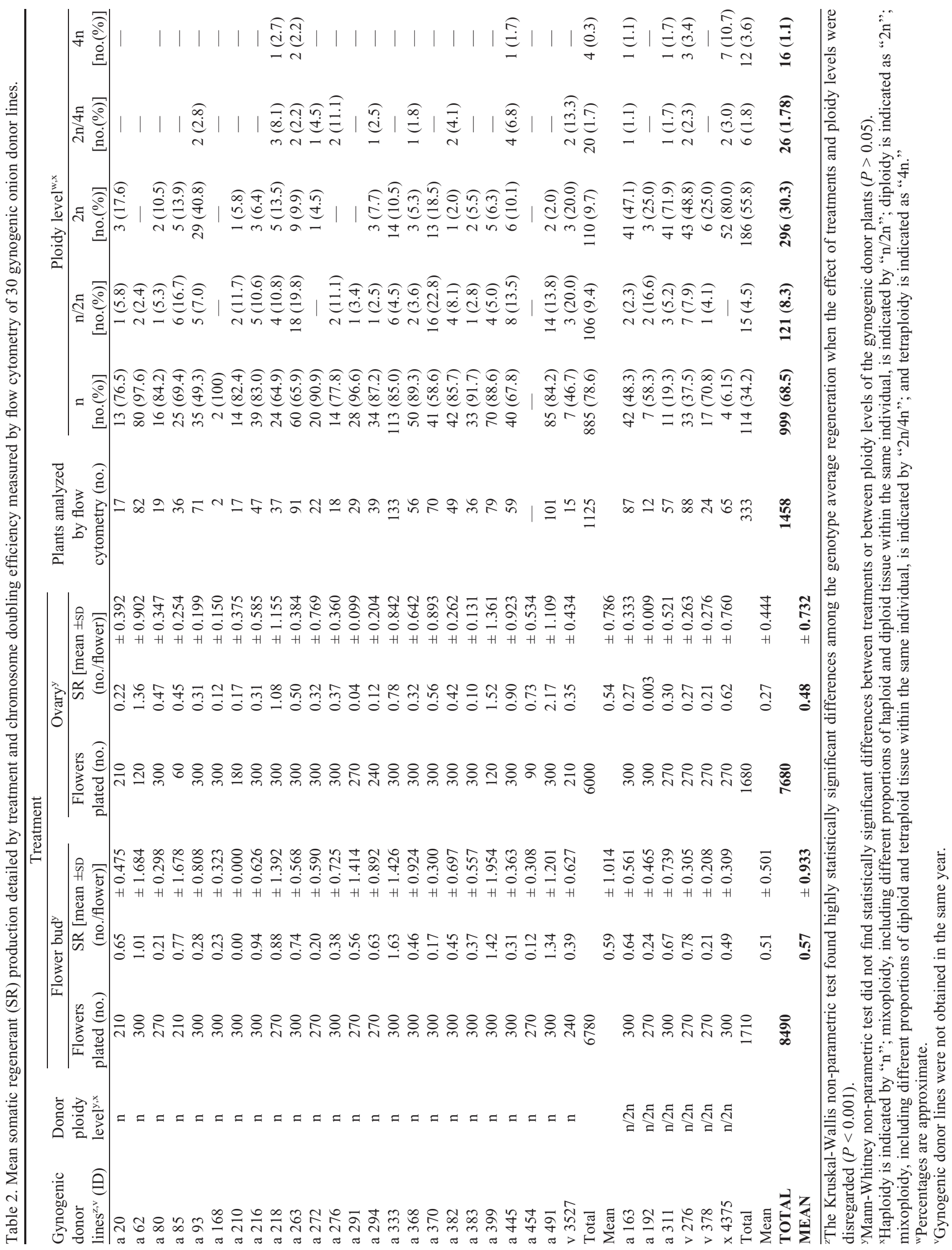


Heterozygous plants within inbred lines may possess a mixture of responsive and nonresponsive genotypic combinations (Javornik et al., 1998). In our studies, during the first cycle of gynogenic induction, haploid regenerants were obtained, but the degree of their gynogenic responsiveness was unknown. To evaluate whether this first generation of haploid regenerants are responsive, a second cycle of gynogenic induction was performed using progenies of selfed DH lines as starting material.

The general decrease in gynogenic responsiveness observed between donors and their selfed DH progenies indicates that gynogenic regeneration may be polygenically controlled (Bohanec et al., 2003). Therefore, a decrease in gynogenic responsiveness among regenerants would be the result of segregation of alleles affecting gynogenesis. However, the environmental effects cannot be ruled out. Some selfed DH donor lines showed an increase in gynogenic responsiveness, therefore, it is possible to select for gynogenic responsiveness as previously reported (Havey and Bohanec, 2007). The results obtained in the present study support the need to select $\mathrm{DH}$ lines according to their gynogenic ability in a second cycle of gynogenesis.

SOMATIC REgENERATION AND CHROMOSOME DOUbling OF HAPLOID PLANTS. The ploidy level of flowering onion plants

Table 3. Pollen viability (percentage of dark red stained pollen grains) of 30 doubled haploid (DH) onion lines.

\begin{tabular}{|c|c|c|c|}
\hline $\begin{array}{l}\text { Inbred source } \\
\text { line (ID) } \\
\end{array}$ & $\begin{array}{l}\text { DH line } \\
(\text { ID })^{z}\end{array}$ & $\begin{array}{c}\text { Pollen cells } \\
\text { surveyed (no.) }\end{array}$ & $\begin{array}{c}\text { Pollen } \\
\text { viability }(\%)\end{array}$ \\
\hline B $0223 \mathrm{~B}$ & x 7608 & 419 & 5.0 \\
\hline B 0223 B & x 1046 & 1749 & 52.9 \\
\hline B 0223 B & x 6923 & 1008 & 54.3 \\
\hline B 0223 B & x 3850 & 951 & 80.0 \\
\hline B 0223 B & x 1217 & 1375 & 82.5 \\
\hline B 1717 A & x 1707 & 81 & 0.0 \\
\hline B 1717 A & у 0137 & 222 & 0.9 \\
\hline B 1717 B x B 2923 B & v 3051 & 822 & 83.2 \\
\hline B 2923 B & x 4375 & 93 & 0.0 \\
\hline B 2923 B & x 3182 & 196 & 3.6 \\
\hline B 2923 B & x 6852 & 264 & 5.7 \\
\hline B 2923 B & x 4576 & 2355 & 12.0 \\
\hline B 2923 B & x 1930 & 1216 & 26.6 \\
\hline B 2923 B & у 0618 & 1765 & 34.7 \\
\hline B 2923 B & x 5333 & 1870 & 37.4 \\
\hline В 2923 B & x 4250 & 853 & 51.3 \\
\hline B 2923 B & x 5198 & 1914 & 55.9 \\
\hline В 2923 B & x 5662 & 780 & 61.5 \\
\hline B 2923 B & x 7018 & 1369 & 68.4 \\
\hline B 2923 B & x 6775 & 2496 & 70.1 \\
\hline B 2923 B & у 1159 & 929 & 74.3 \\
\hline B 2923 B & y 1318 & 1450 & 74.8 \\
\hline B 2923 B & у 0727 & 635 & 80.8 \\
\hline B 2923 B & x 2654 & 1338 & 84.0 \\
\hline B 2923 B & x 0554 & 1399 & 87.2 \\
\hline B 2923 B & x 1854 & 1207 & 90.3 \\
\hline B 2923 B & x 0682 & 1099 & 91.2 \\
\hline B 2923 B & x 6161 & 1708 & 94.0 \\
\hline MSU 2935 B & v 0276 & 759 & 95.0 \\
\hline Timor 135 & r 0047 & 1786 & 96.9 \\
\hline Total & & 34108 & \\
\hline Mean & & & 55,15 \\
\hline
\end{tabular}

${ }^{\mathrm{z}} \mathrm{DH}$ lines were not obtained in the same year. obtained from the second gynogenic cycle was measured using flow cytometry to exclude spontaneous DH plants. Somatic regeneration from immature flowers was performed only from haploid and mixoploid plants that formed morphologically normal and vigorous umbels. The majority of flowers opened from the third to the fifth day of culture on the induction medium. Globular organogenic structures were formed on differentiation medium after $\approx 3$ weeks of culture. As soon as they were observed, these structures were transferred to elongation medium, where shoot clumps were induced until the material was suited to perform ploidy measurements.

Somatic regeneration from haploid and mixoploid plants (Table 2) was very successful; all 30 plants studied were responsive in at least one treatment. A maximum of 1.63 and 2.17 regenerants per plated flower were obtained using the first or second procedure, respectively. The somatic regeneration efficiency of haploid donors was very similar on both treatments, while flower bud culture of mixoploid donors was almost twice as efficient as ovary culture. Whether this response is general or limited to the studied genotypes needs to be further evaluated.

Although continuous flower bud culture was superior to ovary culture regardless of the ploidy level of the donor (mean 0.57 vs. 0.48 regenerants per plated flower), the Mann-Whitney non-parametric test did not find any statistically significant differences $(P$ $>0.05)$ between treatments. The same conclusion was reached when the average regeneration between ploidy levels of the donor plants was analyzed. On the other hand, highly statistically significant differences among average regeneration of genotypes were found by the Kruskal-Wallis non-parametric test $(P<0.001)$.

Regeneration from flower bud culture is less laborious. However, it has been reported to induce callus at the flower base (Luthar and Bohanec, 1999). At least for the studied plants, the frequency of callus formation was low, thus extraction of ovaries seems unjustified. Somatic regeneration frequencies were similar to those previously reported for heterozygous diploid lines (Luthar and Bohanec, 1999). This contrasts with previous attempts (Alan et al., 2007) to induce somatic regeneration from haploid flower buds, which produced much lower yields (up to 0.18 regenerants per flower bud). The higher induction frequency obtained in our studies might be due to the use of a two-step induction/regeneration procedure - characterized by the effect of 2,4-D (auxin) at the induction stage, as well as TDZ (cytokine) at the regeneration stage, and due to the use of gellan gum as gelling agent.

The ploidy level of 1458 somatic regenerants originating from 24 haploid and six mixoploid lines was measured by flow cytometry (Table 2). Chromosome 
doubling efficiency (percentage of diploid regenerants) differed according to the ploidy level of the donor plants. On average, $9.7 \%$ and $55.8 \%$ diploid regenerants were obtained from haploid and mixoploid donor plants, respectively. Although the average diploidization response was low in the haploid lines, four of 24 lines yielded a relatively high proportion of diploid regenerants (around 20\%), showing that this trait might also be genotype dependant. In addition to diploids and haploids, other types of mixoploid and tetraploid plants were recovered, with no apparent pattern in relation to the ploidy level of the gynogenic donor plant. Spontaneous chromosome doubling during adventitious somatic regeneration is a relatively frequent phenomenon. This has been reported in several species, frequently causing the formation of tetraploid regenerants from diploid donors (Chauvin et al., 2003; Meyer et al., 2009; Škof et al., 2007).

Pollen viability of DH lines. Pollen viability was highly variable among the $30 \mathrm{DH}$ lines studied, and ranged from $0 \%$ to $96 \%$ (mean of 55\%) (Table 3). This estimation of male fertility indicates that those DH lines with high pollen viability will be useful as pollinators in hybrid seed production.

The phenomenon of low seed production of some inbred onion lines is well known among onion breeders. However, to our knowledge, data regarding male fertility is scarce in the public domain. High pollen viability was not necessarily associated with gynogenic response because some lines, such as v 3051 and r 0047, have gynogenic responses lower than 2\%, while their pollen viability was over $83 \%$. On the other hand, line $\mathrm{x} 4375$ has a gynogenic responsiveness of $23 \%$, but its pollen viability is very low.
Proposed integrated Method. The goal of a program developing DH lines is to produce at least one DH plant from each haploid regenerant. Our results are very promising because $\mathrm{DH}$ plants were recovered from every single mixoploid $(100 \%)$ and from 20 of 24 haploid (83.3\%) gynogenic donor lines. Based on this fact, and on our previous studies on chromosome doubling using APM (Jakše et al., 2003), an integrated method for establishing homozygous lines has been proposed (Fig. 1).

Haploids are obtained via gynogenesis from selected donor lines. About $10 \%$ of gynogenic haploids spontaneously double to the diploid level (Jakše and Bohanec, 2000). Embryos are treated with APM before transferring them to elongation medium. This treatment can increase chromosome doubling to $\approx 30 \%$, provided embryos are treated for $2 \mathrm{~d}$ in liquid medium with APM in doses varying from 25 to $75 \mu \mathrm{M}$ (Jakše et al., 2003; Grzebelus and Adamus, 2004). Once plantlets are well developed in vitro, they are acclimatized and transplanted directly into 5-L pots. This enables the young onion plant to develop a bulb that will flower after dormancy. Ploidy measurements are performed on leaves or flower buds of flowering plants to identify haploid, diploid, and mixoploid material. Diploids can be directly integrated into the breeding program. Haploids and mixoploids are subjected to somatic regeneration, which is expected to occur primarily from single cells. Once multiple shoots are induced, ploidy measurements on somatic material can be performed. All mixoploid donors are expected to regenerate $\mathrm{DH}$, while the number of haploid donors that will regenerate at least one DH can also be high $(83.3 \%$ in this study, $60 \%$ in Alan et al., 2007). Nevertheless, the response of haploid donors appears to be genotype dependant and the number of recovered DH/haploid

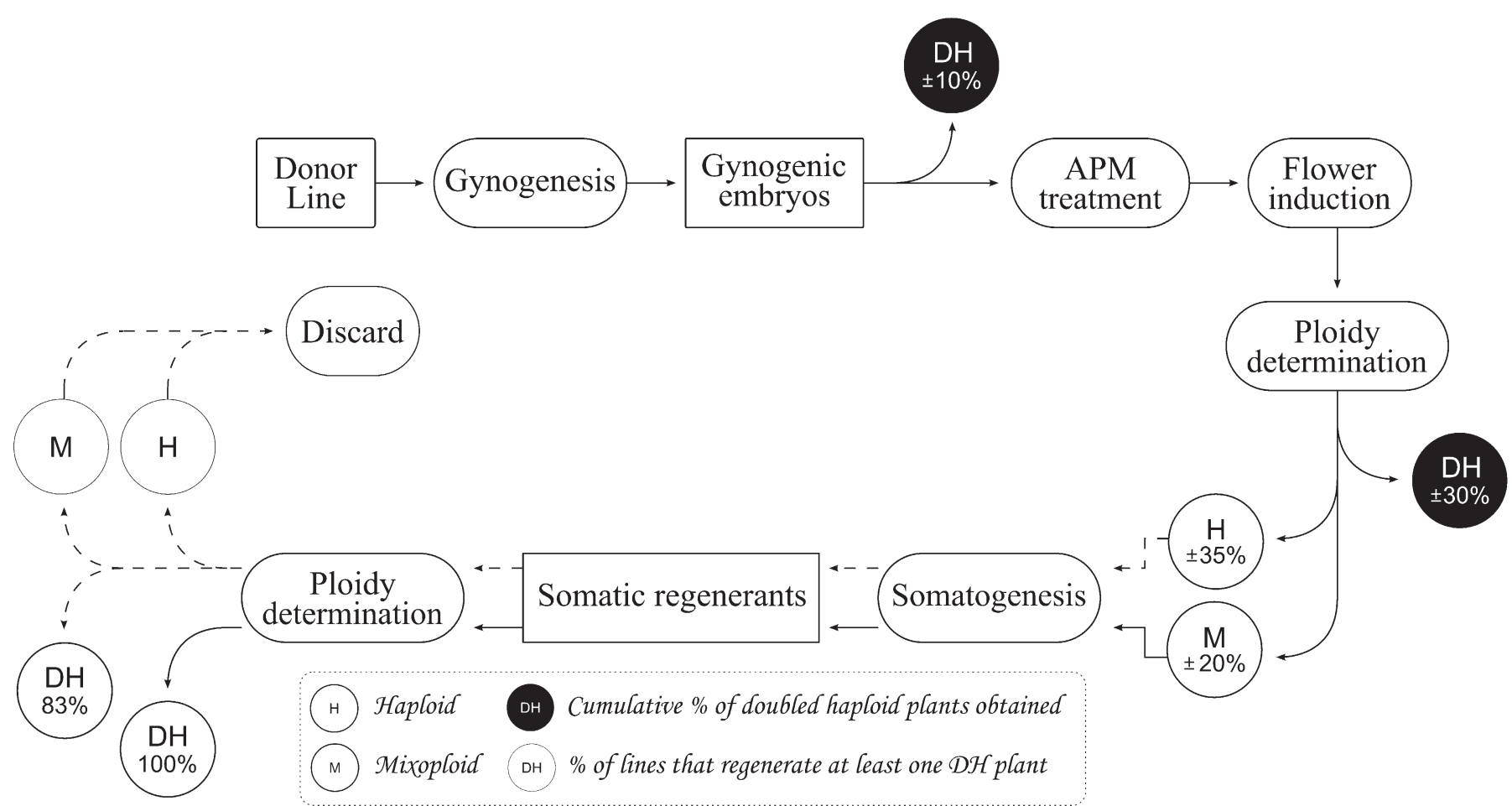

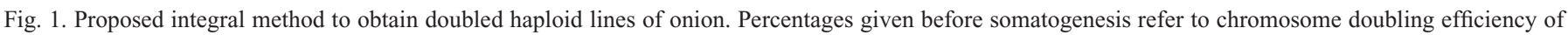
different amiprofos-methyl (APM) treatments published earlier (Jakše et al., 2003). Percentages given after somatic induction refer to the proportion of genotypes (not doubled in the chemical treatment) from which doubled haploids were recovered in this study. Mixoploid in this case refers to different proportions of haploid and diploid tissue within the same individual. 
regenerants can vary widely. Following acclimatization, DH plantlets are ready to be used in the breeding program.

Using this method, the final proportion of haploid donors that will regenerate $\mathrm{DH}$ plantlets can be at least doubled and reach up to around $80 \%$.

Induction of somatic regeneration from flowers of haploids and mixoploids plants allows the successful recovery of these materials that otherwise would be lost for breeding purposes. Direct visual umbel selection before in vitro culture is another advantage of this method because it is highly probable that haploid or mixoploid flowering plants showing normal morphological traits will be fertile once their diploid level is restored. The proposed method minimizes mortality due to the high toxicity of colchicine treatments and due to genotype-dependant response during micropropagation of haploid lines.

\section{Literature Cited}

Alan, A.R., A. Brants, E. Cobb, P.A. Goldschmied, M.A. Mutschler, and E.D. Earle. 2004. Fecund gynogenic lines from onion (Allium cepa L.) breeding materials. Plant Sci. 167:1055-1066.

Alan, A.R., W. Lim, M.A. Mutschler, and E.D. Earle. 2007. Complementary strategies for ploidy manipulations in gynogenic onion (Allium cepa L.). Plant Sci. 173:25-31.

Bohanec, B. 2002. 7. Doubled-haploid onions, p. 145-157. In: H.D. Rabinowitch and L. Currah (eds.). Allium crop science: Recent advances. CABI, Wallingford, UK.

Bohanec, B. and M. Jakše. 1999. Variations in gynogenic response among long-day onion (Allium cepa L.) accessions. Plant Cell Rep. 18:737-742.

Bohanec, B., M. Jakše, and M.J. Havey. 2003. Genetic analyses of gynogenetic haploid production in onion. J. Amer. Soc. Hort. Sci. 128:571-574.

Campion, B. and M. Schiavi. 1994. Production of doubled haploid lines of onion (Allium cepa L.): Progress report and problems. Intl. Colloq. Impact Plant Biotechnol. Agr. Rogla, Slovenia 5-7(June):25-34.

Campion, B., E. Perri, M.T. Azzimonti, E. Vicini, and M. Schiavi. 1995. Spontaneous and induced chromosome doubling in gynogenic lines of onion (Allium cepa L.). Plant Breed. 114:243-246.

Campion, B., M.T. Azzimonti, E. Vicini, M. Schiavi, and A. Falavigna. 1992. Advances in haploid plant induction in onion (Allium cepa L.) through in vitro gynogenesis. Plant Sci. 86:97-104. Chauvin, J.E., C. Souchet, J.P. Dantec, and D. Ellisse'che. 2003. Chromosome doubling of $2 \times$ Solanum species by oryzalin: Method development and comparison with spontaneous chromosome doubling in vitro. Plant Cell Tissue Organ Cult. 73:65-73.

Dunstan, D.I. and K.C. Short. 1977. Improved growth of tissue cultures of onion, Allium cepa. Physiol. Plant. 41:70-72.

Erickson, H.T. and W.H. Gabelman. 1954. Potential value of inbred and $\mathrm{F}_{1}$ hybrid onions for seed production. Proc. Amer. Soc. Hort. Sci. 64:393-398.

Geoffriau, E., R. Kahane, and M. Rancillac. 1997b. Variation of gynogenesis ability in onion (Allium cepa L.). Euphytica 94:37-44. Geoffriau, E., R. Kahane, C. Bellamy, and M. Rancillac. 1997a. Ploidy stability and in vitro chromosome doubling in gynogenic clones of onion (Allium cepa L.). Plant Sci. 122:201-208.
Grzebelus, E. and A. Adamus. 2004. Effect of anti-mitotic agents on development and genome doubling of gynogenic onion (Allium cepa L.) embryos. Plant Sci. 167:569-574.

Hagler, J.R. 1990. Honey bee (Apis mellifera L.) response to simulated onion nectars containing variable sugar and potassium concentrations. Apidologie (Celle) 21:115-121.

Havey, M.J. and B. Bohanec. 2007. Onion inbred line 'B8667 A\&B' and synthetic populations 'Sapporo-Ki-1 A\&B' and 'Onion Haploid1'. HortScience 42:1731-1732.

Jakše, M. and B. Bohanec. 2000. Studies of alternative approaches for genome doubling in onion, p. 101-104. In: B. Bohanec (ed.), COST Action 824, Biotechnological approaches for utilization of gametic cells, Final meeting 1-5 July, Bled, Slovenia. Directorate-General for Research, Communication Unit, European Commission, Brussels, Belgium.

Jakše, M., B. Bohanec, and A. Ihan. 1996. Effect of media components on the gynogenic regeneration of onion (Allium cepa L.) cultivars and analysis of regenerants. Plant Cell Rep. 15:934-938.

Jakše, M., M.J. Havey, and B. Bohanec. 2003. Chromosome doubling procedures of onion (Allium cepa L.) gynogenic embryos. Plant Cell Rep. 21:905-910.

Javornik, B., B. Bohanec, and B. Campion. 1998. Second cycle gynogenesis in onion, Allium cepa L., and genetic analysis of the plants. Plant Breed. 117:275-278.

Keller, E.R.J. and L. Korzum. 1996. 4. Haploidy in onion (Allium cepa L.) and other Allium species, p. 51-75. In: S.M. Jain, S.K. Sopory, and R.E. Veilleux (eds.). In vitro haploid production in higher plants. Vol. 3. Kluwer Academic Publishers, Dordrecht, The Netherlands.

Lederhouse, R.C., D.M. Caron, and R.A. Morse. 1968. Onion pollination in New York. New York's Food. Life Sci. 1:8-10.

Luthar, Z. and B. Bohanec. 1999. Induction of direct somatic organogenesis in onion (Allium cepa L.) using a two-step flower or ovary culture. Plant Cell Rep. 18:797-802.

Martínez, L.E., C.B. Agüero, M.E. López, and C.R. Galmarini. 2000. Improvement of in vitro gynogenesis induction in onion (Allium cepa L.) using polyamines. Plant Sci. 156:221-226.

Meyer, E.M., D.H. Touchell, and T.G. Ranney. 2009. In vitro shoot regeneration and polyploid induction from leaves of Hypericum species. HortScience 44:1957-1961.

Michalik, B., A. Adamus, and E. Nowak. 2000. Gynogenesis in Polish onion cultivars. J. Plant Physiol. 156:211-216.

Muren, R.C. 1989. Haploid plant induction from unpollinated ovaries in onion. HortScience 24:833-834.

Nye, W.P. and N.D. Waters. 1971. Factors affecting pollination of onion in Idaho during 1969. J. Amer. Soc. Hort. Sci. 96:330-332.

Ockendon, D.J. and P.J. Gates. 1976. Variation in pollen viability in onion (Allium cepa L.). Euphytica 25:753-759.

Ponce, M., L. Martínez, and C. Galmarini. 2006. Influence of CCC, putrescine and gellam gum concentration on gynogenic embryo induction in Allium cepa. Biol. Plant. 50:425-428.

Škof, S., B. Bohanec, D. Kastelec, and Z. Luthar. 2007. Spontaneous induction of tetraploidy in hop using adventitious shoot regeneration method. Plant Breed. 126:416-421.

Stöldt, A. 1994. Untersuchungen zur In-vitro-Polyploidisierung von Allium sp. M.Sc. Thesis, Univ. Hamburg, Hamburg, Germany.

Villanueva-Mosqueda, E. and M.J. Havey. 2001. Genetic analyses of seed yield in onion. J. Amer. Soc. Hort. Sci. 126:575-578. 